\title{
Trapped and Escaping Orbits in an Axially Symmetric Galactic-Type Potential
}

\author{
Euaggelos E. Zotos \\ Department of Physics, Section of Astrophysics, Astronomy and Mechanics, \\ Aristotle University of Thessaloniki, GR-541 24, Thessaloniki, Greece \\ Email: evzotos@astro.auth.gr
}

\begin{abstract}
In the present article, we investigate the behavior of orbits in a time-independent axially symmetric galactic-type potential. This dynamical model can be considered to describe the motion in the central parts of a galaxy, for values of energies larger than the energy of escape. We use the classical surfaceof-section method in order to visualize and interpret the structure of the phase space of the dynamical system. Moreover, the Lyapunov characteristic exponent is used in order to make an estimation of the degree of chaoticity of the orbits in our galactic model. Our numerical calculations suggest that in this galactic-type potential there are two kinds of orbits: (i) escaping orbits and (ii) trapped orbits, which do not escape at all. Furthermore, a large number of orbits of the dynamical system display chaotic motion. Among the chaotic orbits, there are orbits that escape quickly and also orbits that remain trapped for vast time intervals. When the value of a test particle's energy slightly exceeds the energy of escape, the number of trapped regular orbits increases as the value of the angular momentum increases. Therefore, the extent of the chaotic regions observed in the phase plane decreases as the energy value increases. Moreover, we calculate the average value of the escape period of chaotic orbits and try to correlate it with the value of the energy and also with the maximum value of the $z$ component of the orbits. In addition, we find that the value of the Lyapunov characteristic exponent corresponding to each chaotic region for different values of energy increases exponentially as the energy increases. Some theoretical arguments are presented in order to support the numerically obtained outcomes.
\end{abstract}

Keywords: Galaxies: kinematics and dynamics

Received 2012 January 19, accepted 2012 March 4, published online 2012 April 4

\section{Introduction}

The shape of galaxies strongly depends on their orbital populations. Stars within galaxies belong to orbital families. In particular, the size and the shape of a galaxy determines the relative populations of these families. As a galaxy evolves, capture and escape of stars between these families takes place. Therefore, capture and escape are generic processes that will have occurred many times in the lifetime of a galaxy.

Each orbital family has a parent periodic orbit, that is an orbit that describes a closed figure. For example, in a spherical galaxy all stars belong to the family of tube orbits, members of which librate around closed circular orbits. An oval distortion in the center of the galaxy is supported by the family of box orbits, the parent periodic orbits of which are radial orbits. As the distortion grows, stars are transferred from the loop to the box orbital family. This capture process is important in the formation, maintenance and secular evolution of non-axisymmetric structure, such as galactic bars, rings and spiral arms (Kalnajs 1973; Contopoulos, Hénon \& Lynden-Bell 1973; Tremaine \& Weinberg 1984). Whether or not a trapped star remains trapped may depend on the presence of a central massive black hole or a simple mass concentration. Close encounters can scatter stars away from their initial orbits and thus cause a gradual disruption of the population of the orbits.

The phenomenon of escaping stars from stellar systems has been an active field of research during the last decades (Contopoulos 1990; Contopoulos \& Kaufmann 1992; Siopis et al. 1995a; Siopis, Contopoulos \& Kandrup 1995b; Kandrup et al. 1999; Fukushige \& Heggie 2000; Contopoulos \& Efstathiou 2004; Contopoulos \& Harsoula 2005; Contopoulos \& Patsis 2006; Papadopoulos \& Caranicolas 2007). The reader can find more details on the subject of escapes in the review of Siopis et al. (1996) and also in the book of Contopoulos (2002). When a star has a value of energy higher than the energy of escape, the equipotential curves or, equivalently, the zero-velocity curves (ZVCs) are open and there are cases in which a star can escape from the stellar system. On the other hand, there are also cases in which stars that hold values of energy much larger than the energy of escape do not escape at all, even though the ZVCs are open and therefore at least one channel of escape exists. A characteristic example of such non-escaping stars 
comprises those moving in orbits with initial conditions close to those of a stable periodic orbit. Apart from galactic dynamics, the phenomenon of escaping and trapped orbits has also been studied in the case of the three-body problem (Anosova 1986; Benet, Trautmann \& Seligman 1996; Benet, Seligman \& Trautman 1998), as well as in mappings (Bleher et al. 1988).

The main scope of the present research is to shed light on the properties of motion in a dynamical system with trapped and escaping orbits. In particular, we shall study the nature of motion (regular or chaotic) for values of energy larger than the energy of escape, that is the case when the ZVC is open. In this case, there are regular orbits that do not escape at all. We shall give the set of initial conditions leading to this kind of orbit as a function of the energy. What is much more interesting is that there are two different kinds of chaotic orbits. The first kind of chaotic orbits consists of chaotic orbits that remain trapped inside the ZVC for long time intervals before escaping to infinity. We shall call these orbits trapped chaotic orbits and investigate their properties, such as their time-scale of escape and their degree of chaos. The second kind of chaotic orbits consists of chaotic orbits that escape from the ZVC in very short time intervals. These orbits are called fast-escaping orbits. The main feature of these orbits is that their initial conditions are very close to the neighborhood of the escape channel of the ZVC.

The layout of this article is as follows. In Section 2 we present a mathematical analysis of our galactic-type model and also a numerically obtained relationship connecting the angular momentum $L_{z}$ and the energy of escape $h_{\text {esc }}$. Moreover, in the same section we study the areas of initial conditions corresponding to trapped regular orbits as a function of the energy. In Section 3, we study the structure of the dynamical system using the outcomes from the phase planes. A numerical relationship between the extent of the chaotic regions and the value of the energy is also presented. In Section 4 we conduct a detailed study on trapped and escaping chaotic orbits and we also connect the degree of chaos with the value of the energy. We close with Section 5, where a discussion and the conclusions of this research are given and a comparison with previous work is also made.

\section{Description of the Dynamical Model}

Here we shall study the properties of motion in the galactic-type potential

$$
V(r, z)=\frac{\omega^{2}}{2}\left(r^{2}+z^{2}\right)-\epsilon\left[\alpha\left(r^{4}+z^{4}\right)+2 \beta r^{2} z^{2}\right] .
$$

Potentials of the form of Equation 1, made up of perturbed harmonic oscillators, are among the most well-studied in non-linear dynamics (see Deprit \& Elipe 1991; Elipe \& Deprit 1999; Elipe 2001). Moreover, this potential has been applied several times in previous research works (Caranicolas \& Vozikis 1999; Caranicolas 2001; Karanis \& Caranicolas 2002; Caranicolas \& Vozikis
2002; Caranicolas \& Papadopoulos 2003b) and can be derived by expanding global galactic potentials near the central stable equilibrium point of the system, that is the center of the galaxy. In the present study the above potential can be considered to describe the local motion in the meridian $(r, z)$ plane near the central parts of an axially symmetric galaxy. In Equation $1,(r, z)$ are the usual cylindrical coordinates, while $\omega, \epsilon, \alpha$, and $\beta$ are parameters. Such galactic-type potentials arise naturally if (a) the density distribution near the galactic center is an analytic function of the coordinates and (b) the Taylor series for the corresponding potential is truncated at fourth order. Here we must point out that our gravitational galactic-type potential is truncated at $r_{\max }=1.5$ otherwise the mass density increases outwards from the center, which is almost never observed in galaxies or other stellar systems. Therefore, we study the phenomenon in which stars escape from the central parts of a galaxy.

It is well known that the motion of stars in the central parts of almost all galaxies could be satisfactorily represented by the motion of a harmonic oscillator. Therefore, our dynamical model of the perturbed harmonic oscillator potential given by Equation 1 is able to describe the local motion of stars in the central region of different kinds of galaxies (i.e. from a non-rotating elliptical galaxy to a disk or spiral galaxy). Once more, we have to point out that the present model can only describe local motion at small distances from the galactic center $\left(r_{\max } \leq 1.5 \mathrm{kpc}\right)$.

As the potential $V(r, z)$ is axially symmetric and the $L_{z}$ component of angular momentum is conserved, the dynamical structure of the galactic system can be investigated using the effective potential

$$
V_{\mathrm{eff}}(r, z)=\frac{L_{z}^{2}}{2 r^{2}}+V(r, z)
$$

in order to study the properties of motion in the meridian $(r, z)$ plane. The equations of motion are

$$
\begin{array}{rlrl}
\dot{r} & =p_{r}, & \dot{z} & =p_{z}, \\
\dot{p}_{r}=-\frac{\partial V_{\text {eff }}}{\partial r}, & \dot{p}_{z}=-\frac{\partial V_{\text {eff }}}{\partial z},
\end{array}
$$

where the dot indicates the derivative with respect to time.

The Hamiltonian corresponding to the potential of Equation 2 is written as

$$
H=\frac{1}{2}\left(p_{r}^{2}+p_{z}^{2}\right)+V_{\mathrm{eff}}(r, z)=h,
$$

where $p_{r}$ and $p_{z}$ are the momenta per unit mass conjugate to $r$ and $z$ respectively, while $h$ is the numerical value of the Hamiltonian, which is conserved. Equation 4 is an integral of motion, which indicates that the total energy of the test particle is conserved. The Hamiltonian of Equation 4 also describes the motion in the $(r, z)$ meridian plane, rotating at the angular velocity

$$
\dot{\phi}=\frac{L_{z}}{r^{2}} .
$$




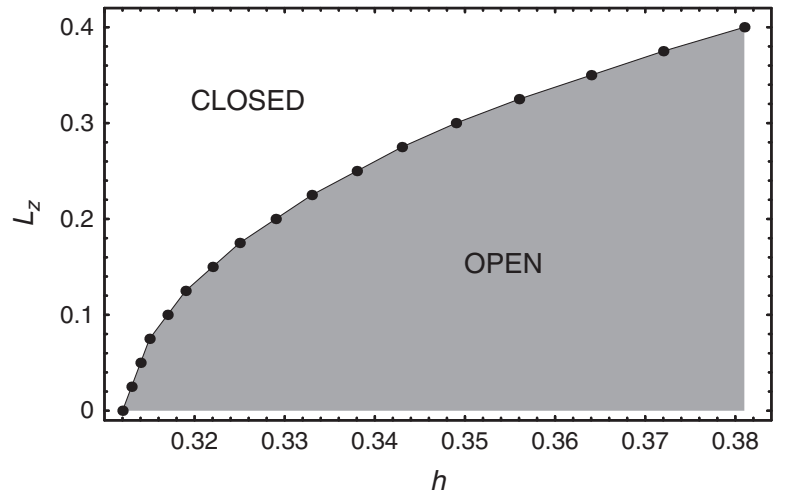

Figure 1 A plot of the correlation between $L_{z}$ and $h$. In the upper left part of the $\left[h, L_{z}\right]$ plane, including the solid line, the ZVC is closed, while in the lower shaded right part the $\mathrm{ZVC}$ is open.

The bulk of the outcomes of present research are based on numerical integration of the equations of motion (Equation 3). We use a Bulirsh-Stöer integration routine in FORTRAN 95, with double precision in all subroutines. The accuracy of our results was checked by the constancy of the value of the energy integral (Equation 4), which was conserved up to the fifteenth significant figure.

In this work we use a system of galactic units, where the unit of length is $1 \mathrm{kpc}$ and the unit of time is $10^{7} \mathrm{yr}$. The velocity unit is $1 \mathrm{kpc}\left(10^{7} \mathrm{yr}\right)^{-1}=97.8 \mathrm{~km} \mathrm{~s}^{-1}$ and the energy unit is $1 \mathrm{kpc}^{2}\left(10^{7} \mathrm{yr}\right)^{-2}$. In these units, we take the values $\omega=1\left(10^{7} \mathrm{yr}\right)^{-1}, \epsilon=1\left(10^{7} \mathrm{yr} \mathrm{kpc}\right)^{-2}, \alpha=0.2$, and $\beta=-1.2$. The above numerical values of the quantities of the dynamical system are kept constant during this research; they secure positive density everywhere, free of singularities.

In the case when $L_{z}=0$, the energy of escape for the potential in Equation 1 can be found theoretically (see Caranicolas \& Vozikis 1999 and references therein) and is equal to

$$
h_{\mathrm{esc}}=\frac{1}{16 \epsilon \alpha} .
$$

When $\alpha=0.2, \beta=-1.2$, and $\epsilon=1$, we find $h_{\text {esc }}=0.3125$. In the general case where $L_{z} \neq 0$, the value of the energy of escape can be found numerically. Figure 1 shows a plot of the relation between the angular momentum $L_{z}$ and the energy $h$. In the upper left part of the $\left[h, L_{z}\right]$ plane, including the line, the ZVC is closed and the motion of stars is bounded, while in the lower shaded right part of the same diagram the ZVC is open and therefore stars can escape through the open channel. The relation between $h_{\text {esc }}$ and $L_{z}$ is given approximately by the fourth-order polynomial equation

$$
\begin{aligned}
L_{z}= & -55738.3 h_{\mathrm{esc}}^{4}+78897.2 h_{\mathrm{esc}}^{3}-41862.2 h_{\mathrm{esc}}^{2} \\
& +9871.87 h_{\mathrm{esc}}-872.985 .
\end{aligned}
$$

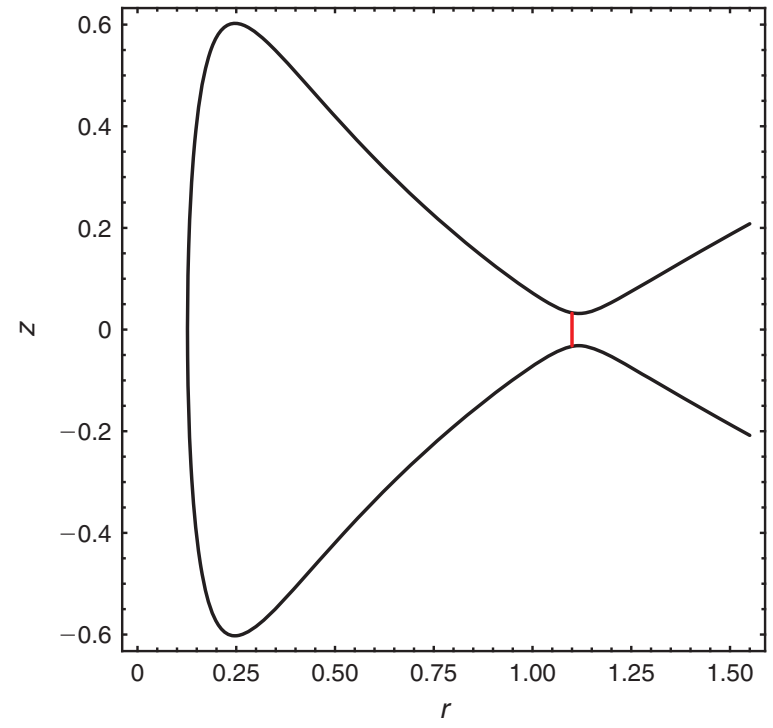

Figure 2 The ZVC when $h=0.32$ and $L_{z}=0.10$. The vertical (red) line represents the Lyapunov orbit.

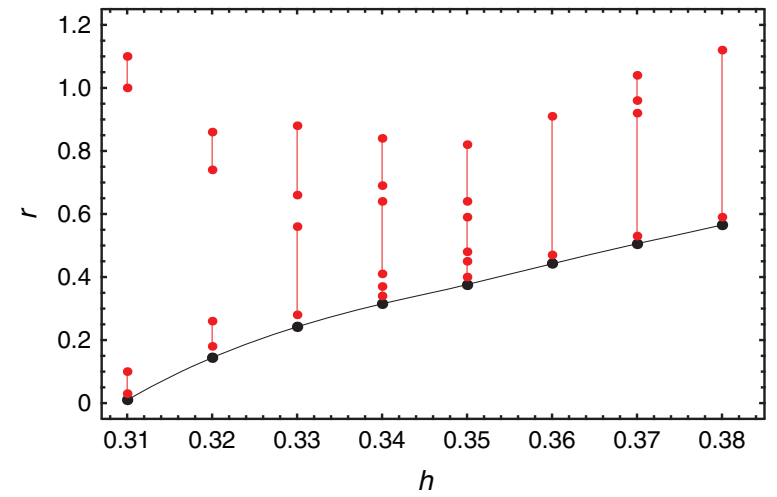

Figure 3 The black solid line depicts the relationship between $r_{\text {min }}$ and $h$, while the vertical (red) lines correspond to intervals of initial conditions on the $r$ axis of the phase plane corresponding to trapped regular orbits. See text for details.

Figure 2 shows the ZVC when $h=0.32$ and $L_{z}=0.10$. As one can see, there is a channel in the ZVC through which orbits can escape to infinity. The vertical (red) line represents the Lyapunov orbit. Orbits that cross the Lyapunov orbit with velocity outwards will escape from the dynamical system (Churchill et al. 1979).

In addition to the escaping orbits, there are regular orbits that do not escape at all. The vertical (red) lines in Figure 3 show intervals corresponding to initial conditions on the $r$ axis of the $\left(r, p_{r}\right)$ phase planes $(z=0)$ that give such trapped regular orbits, for each particular value of the energy $h$. We select pairs of $\left[h, L_{z c}\right]$ in the following way, We take a pair of values from Equation 7, say $\left[h, L_{z c}\right]=[0.32,0.11]$. Then we choose the pair $h=h_{\mathrm{esc}}$, $L_{z}=L_{z c}-\Delta L$ with $\Delta L=0.01$. For this pair of values the ZVC is always open. The solid line in Figure 3 gives the values of $r_{\mathrm{min}}$, that is the intersection of the $\mathrm{ZVC}$ 

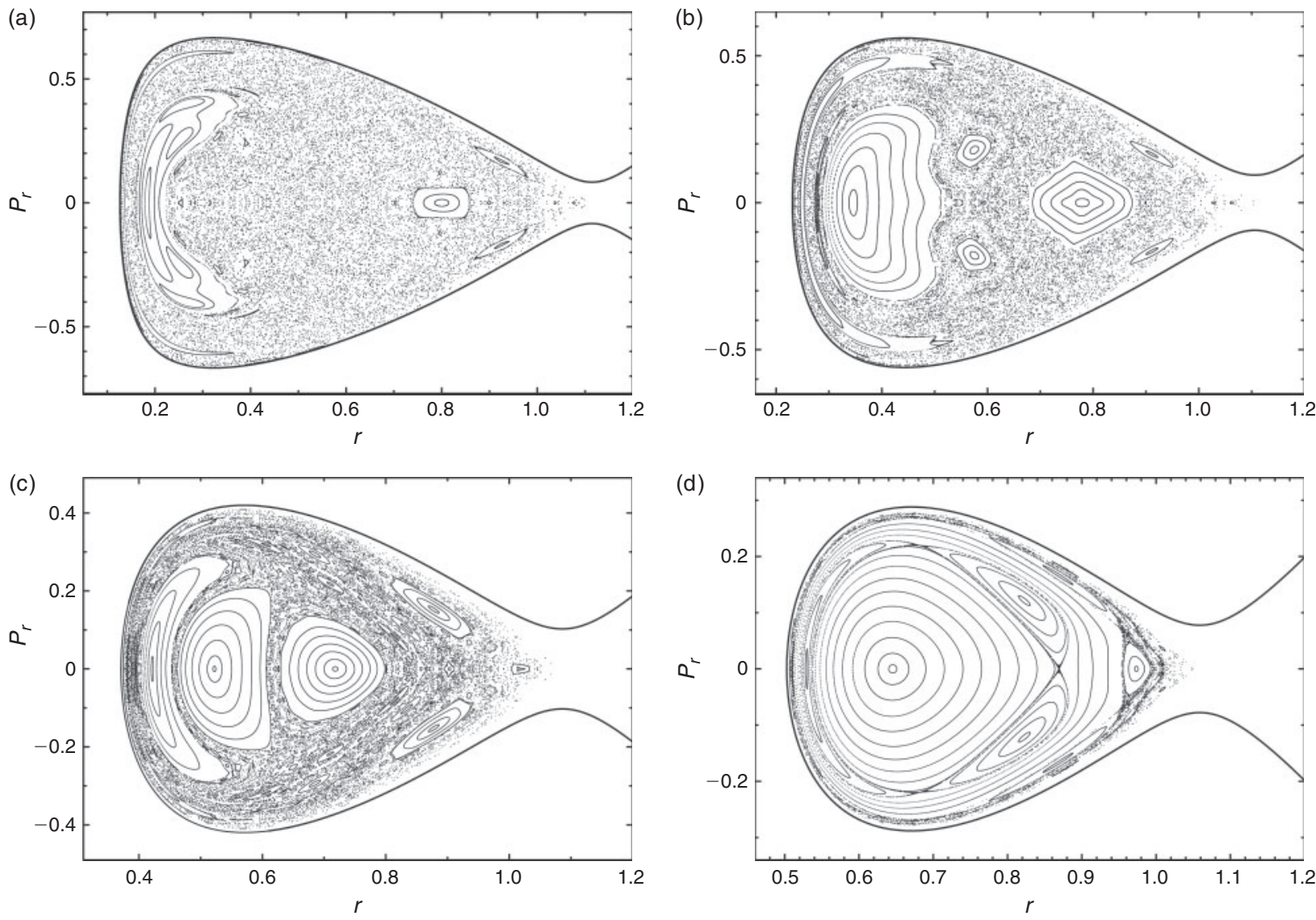

Figure 4 The $\left(r, p_{r}\right), z=0, p_{z}>0$ phase plane for the Hamiltonian (4) when (a) $h=0.32$ and $L_{z}=0.10$, (b) $h=0.33$ and $L_{z}=0.18$, (c) $h=0.35$ and $L_{z}=0.28$ and (d) $h=0.37$ and $L_{z}=0.36$.

with the $z=0$ axis. This can be found if we solve numerically the equation

$$
V_{\mathrm{eff}}(r)=V(r)+\frac{L_{z}^{2}}{2 r^{2}}=\frac{1}{2} r^{2}-\alpha r^{4}+\frac{L_{z}^{2}}{2 r^{2}}=h
$$

We find that the value of $r_{\min }$ increases as the value of the energy $h$ increases. This means that as the energy increases orbits move far from the center. In fact, it is true that, as $h_{\text {esc }}$ and $L_{z}$ are connected through Equation 7, orbits with larger $L_{z}$ are not able to approach near the center. It is clear from the diagram of Figure 3 that the area of the initial conditions on the $r$ axis of the $\left(r, p_{r}\right)$ phase planes, that is initial conditions $\left(r=r_{0}, z=z_{0}=0\right)$, that give regular trapped orbits generally increases as the value of energy $h$ increases. We shall explain this point in detail later in the next section, when we will try to connect the extent of the chaotic regions of the phase planes with the value of the energy $h$.

\section{Structure of the Dynamical System}

In this section, we use a qualitative but very effective method to reveal the dynamical structure of a Hamiltonian system. In this method, we plot the successive intersections of the orbits using the $\left(r, p_{r}\right), z=0, p_{z}>0$ surface of section, in order to determine the regular or chaotic nature of the motion. This particular method has been extensively applied to Hamiltonian systems with two degrees of freedom, as in these systems the phase plane is a two-dimensional plane and therefore can easily be visualized and interpreted. Here we must note that this phase plane is not a Poincare surface of section, because some orbits have loops that may or may not intersect this surface. A Poincaré surface of section exists only if orbits intersect a surface like $z=0$ at least once within a certain time interval. However, escaping orbits in general do not intersect the $z=0$ axis after a certain time; thus the sections of the present paper are not Poincare sections. If we set $z=p_{z}=0$ in Equation 4, we obtain the limiting curve in the $\left(r, p_{r}\right)$ phase plane, which is the curve containing all the invariant curves for a given value of the energy integral $h$. Thus we choose the values of $r$ and $p_{r}$ inside the limiting curve, while the value of $p_{z}$ is found always from the energy integral (4). The limiting curve of the dynamical system corresponds to

$$
\frac{1}{2} p_{r}^{2}+V_{\mathrm{eff}}(r)=h
$$

Figure 4 shows the $\left(r, p_{r}\right), z=0, p_{z}>0$ surfaces of section for the Hamiltonian of Equation 4, obtained by numerical integration of the equations of motion (Equation 3). In all cases the outermost solid curves, which are the ZVCs, are open. The values of energy $h$ and angular 
momentum $L_{z}$ are chosen as described in the previous section. In Figure 4(a), we have the case in which $h=0.32$ and $L_{z}=0.10$. As one can see, the majority of the phase plane is covered by chaotic orbits, producing a unified chaotic sea, while there are also areas of regular motion. In addition to the above, one can also observe smaller islands of invariant curves embedded in the chaotic sea, which are produced by secondary resonant orbits. Figure 4(b) shows the $\left(r, p_{r}\right)$ phase plane when $h=0.33$ and $L_{z}=0.18$. In this case the area in the phase plane occupied by regular orbits is larger than in Figure 4(a), while the resonant islands look more prominent. Figure 4(c) shows the $\left(r, p_{r}\right)$ phase plane when $h=0.35$ and $L_{z}=0.28$. Here the extent of the chaotic area has decreased and the structure of the phase plane is very complicated and interesting as well. We observe that, apart from the invariant curves corresponding to the basic families of orbits of the dynamical system, numerous small embedded islands of invariant curves have appeared, produced by complicated secondary resonant orbits. We will study these secondary resonant orbits later in the next section. Figure $4(d)$ shows the $\left(r, p_{r}\right)$ phase plane when $h=0.37$ and $L_{z}=0.36$. In this case, the situation is quite different from that in the previous phase planes. Here the majority of orbits are regular, while a very small chaotic layer, which is confined in the outer parts of the phase plane, is present. Secondary resonances are still observed.

The main conclusions from the above analysis of the $\left(r, p_{r}\right)$ phase planes of the dynamical system are as follows.

(i) The area in the phase planes occupied by regular orbits increases significantly as the value of the energy increases. At the same time, chaotic regions are reduced with increasing value of energy $h$. Our numerical calculations indicate that the area corresponds to regular orbits in the $\left(r, p_{r}\right)$ phase planes and increases as we proceed to larger values of $h$ and $L_{z}$, always following Equation 7 and the same way of choosing the pairs $\left[h, L_{z}\right]$ as has been described in the previous section.

(ii) As the value of energy $h$ increases, we observe that the maximum velocity $p_{r}$ in the $\left(r, p_{r}\right)$ phase planes decreases. Furthermore, the whole area of the phase planes is reduced and becomes smaller as the value of energy $h$ increases.

(iii) The relative width of the channel in the ZVC through which orbits can escape to infinity increases as the values of energy $h$ and angular momentum $L_{z}$ increase. Therefore, one may conclude that as we move to larger values of $h$ and $L_{z}$ the probability of a star escaping from the dynamical system increases.

Figure 5 shows a plot of the relationship between the percentage of area $A \%$ covered by chaotic orbits in the $\left(r, p_{r}\right)$ phase plane and the energy of the dynamical system $h$. We see that the percentage $A \%$ decreases as the value of energy $h$ increases. Note that for small values of $h$ the slope of the curve in the diagram is small, while for larger

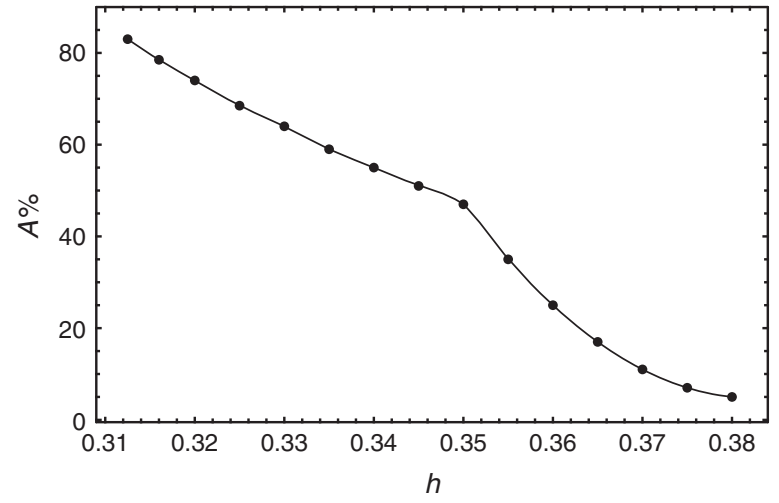

Figure 5 A plot of the relationship between the percentage of the area $A \%$ covered by chaotic orbits in the $\left(r, p_{r}\right)$ phase plane and the value of the energy $h$ of the dynamical system.

values of the energy $(h \geq 0.35)$ the slope is high. We must point out that the percentage $A \%$ is calculated as follows: we choose 1000 orbits with different and random initial conditions $\left(r_{0}, p_{r 0}\right)$ in each $\left(r, p_{r}\right)$ phase plane and then we take the ratio of the number of those that produce chaotic orbits to the total number of tested orbits (see Zotos 2011a, 2011b). Here we must remember that the higher $h$, the higher $L_{z}$ (see Figure 1). Therefore we have a different slope for small values of the angular momentum $L_{z}$. This phenomenon is similar to that observed in studies connecting chaos to angular momentum (Caranicolas \& Innanen 1991; Caranicolas \& Papadopoulos 2003a; Caranicolas \& Zotos 2010; Caranicolas \& Zotos 2011b; Zotos 2011a, 2011b).

\section{Analysis of Trapped and Escaping Orbits}

Let us now proceed to study the various families of regular orbits that appear in our dynamical model. Figure 6 shows four periodic orbits that represent the four basic families of orbits of this galactic system. By the term 'basic orbits' of the dynamical system, we mean resonant orbits of low multiplicity (such as the orbits shown in Figure 6). Figure 6(a) shows an orbit with initial conditions $r_{0}=0.8, z_{0}=0, p_{r 0}=0$, while the value of $p_{z 0}$ is found from the energy integral (Equation 4 ) for all orbits. This orbit is a typical example of the $1: 1$ resonance. The values of the energy $h$ and angular momentum $L_{z}$ are as in Figure 4(a). In Figure 6(b) we see a regular orbit, characteristic of the $2: 1$ resonance. This orbit has initial conditions $r_{0}=0.1832, z_{0}=0, p_{r 0}=0$, while the values of the other parameters are as in Figure 4(a). Figure 6(c) depicts a periodic orbit with initial conditions $r_{0}=0.925$, $z_{0}=0, p_{r 0}=0.169$. This orbit characterizes the family of $2: 3$ resonant orbits. The values of energy and angular momentum for this orbit are as in Figure 4(a). In Figure 6(d) we can observe a typical example of a $4: 3$ resonant orbit, which has initial conditions $r_{0}=0.2757$, $z_{0}=0, p_{r 0}=0$. For this orbit the values of $h$ and $L_{z}$ are as in Figure 4(b). All orbits were calculated for a time period of 100 time units. 
(a)

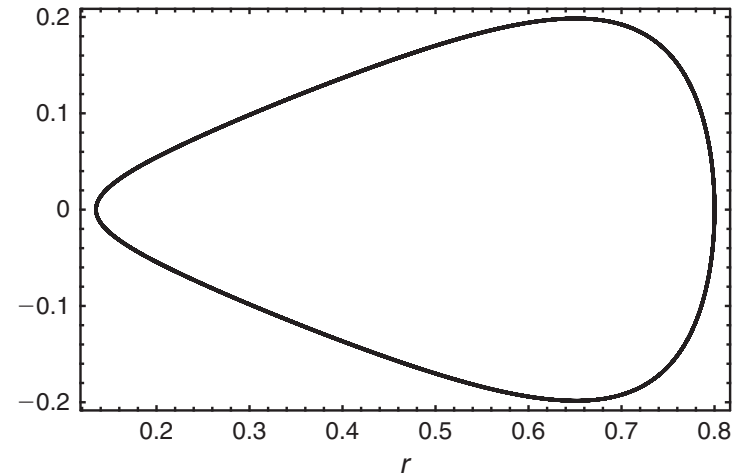

(c)

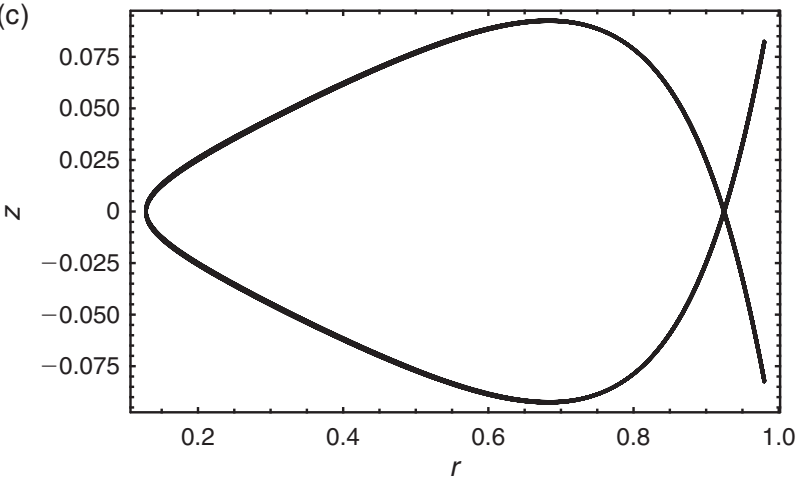

(b)

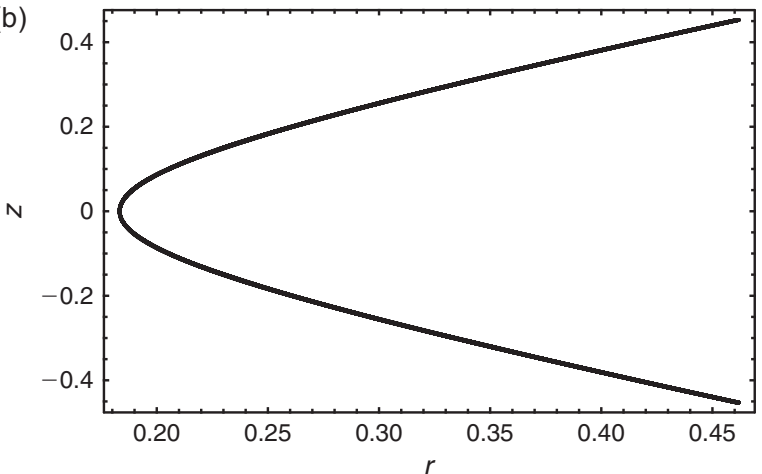

(d)

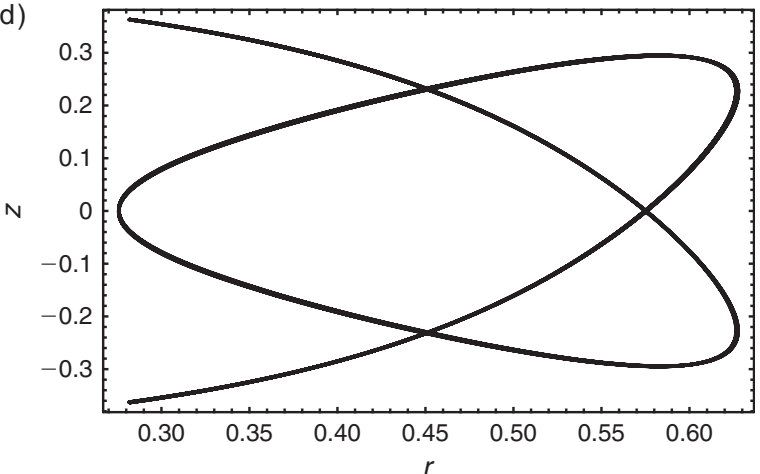

Figure 6 Four periodic orbits of the dynamical system. (a) $1: 1$ resonant periodic orbit, (b) $2: 1$ resonant periodic orbit, (c) $2: 3$ resonant periodic orbit and (d) $4: 3$ resonant periodic orbit. More details and the initial conditions of the orbits are given in the text.

Apart from the families of basic periodic orbits, as we can see from the phase planes shown in Figure 4 the dynamical system contains numerous and more complicated periodic orbits produced by secondary resonances. These secondary resonances correspond to multiple islands of invariant curves in the $\left(r, p_{r}\right)$ phase planes. Figure 7 shows eight secondary resonant periodic orbits of the dynamical system. Figure 7(a) shows a resonant orbit with initial conditions $r_{0}=0.3908, z_{0}=0, p_{r 0}=0$, while the values of the energy and the angular momentum are as in Figure 4(c). In Figure 7(b) we can see a periodic orbit that has initial conditions $r_{0}=0.39549, z_{0}=0$, $p_{r 0}=0$. The values for all the other parameters are as in Figure 4(c). Figure 7(c) depicts a resonant orbit with initial conditions $r_{0}=1.0627, z_{0}=0, p_{r 0}=0$, while the values of $h$ and $L_{z}$ are as in Figure 4(a). In Figure 7(d), one can observe an orbit with initial conditions $r_{0}=0.7027$, $z_{0}=0, p_{r 0}=0$. For this orbit the values of the energy and the angular momentum are as in Figure 4(a). Figure 7(e) shows a secondary resonant orbit with initial conditions $r_{0}=0.2543, z_{0}=0, p_{r 0}=0$. The values for all other parameters are as in Figure 4(a). In Figure 7(f), one can see a periodic complicated orbit that has initial conditions $r_{0}=0.8574, z_{0}=0, p_{r 0}=0$. The values of $h$ and $L_{z}$ are as in Figure 4(c). A much more complicated orbit with initial conditions $r_{0}=0.1597, z_{0}=0, p_{r 0}=0$ is shown in Figure $7(\mathrm{~g})$. The values of the energy and the angular momentum for this orbit are as in Figure 4(a). Finally, in Figure 7(h) we can observe a resonant periodic orbit that has initial conditions $r_{0}=0.39692, z_{0}=0, p_{r 0}=0$, while the values for all other parameters for this orbit are as in
Figure 4(c). All resonant periodic orbits shown in Figure 7 were calculated for a time period of 100 time units. It is quite interesting to note that a simple dynamical model such as the one used in this present research is able to produce so many secondary resonant orbits.

Here, we must note that periodic orbits are an important factor of internal dynamical evolution in galaxies. Romero-Gómez et al. (2011) computed the orbits confined by the invariant manifolds of the unstable periodic orbits located at the ends of the bar of the Milky Way. Moreover, the chaotic orbits by the invariant manifolds associated with periodic orbits affect the barred and spiral structure of a galaxy. Furthermore, bars in galaxies are mainly supported by particles trapped around stable periodic orbits. These orbits represent oscillatory motion with only one frequency, the bar-driving frequency, and miss free oscillations. Maciejewski \& Athanassoula (2007) revealed that a similar situation takes place in double bars: particles become trapped around parent orbits, which in this case represent oscillatory motion with two frequencies of driving by the two bars and which also lack free oscillations. Thus the parent orbits, which constitute the backbone of an oscillating potential of two independently rotating bars, are double-frequency orbits. These orbits do not close in any reference frame, but they map on to closed curves called loops. Trajectories trapped around the parent double-frequency orbit map on to a set of points confined within a ring surrounding the loop. The families of periodic orbits in a galaxy may affect spiral arms or thick disks as well as the barred structure. 
(a)

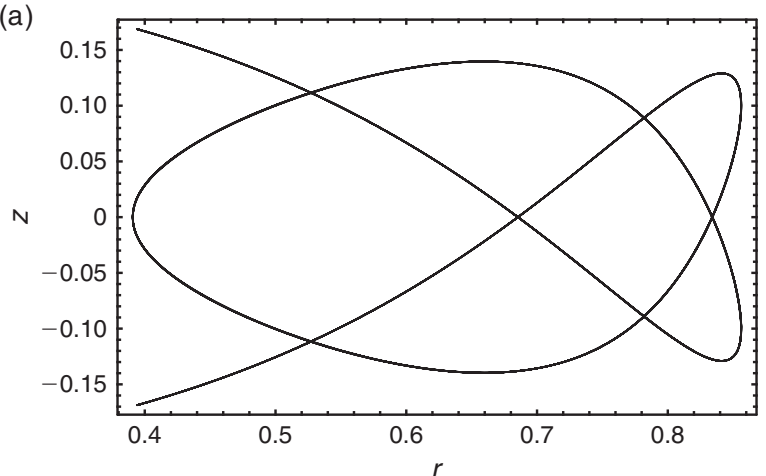

(c)

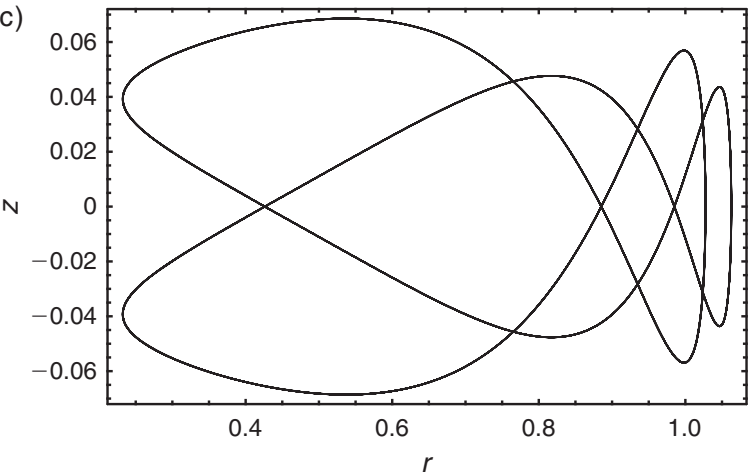

(e)

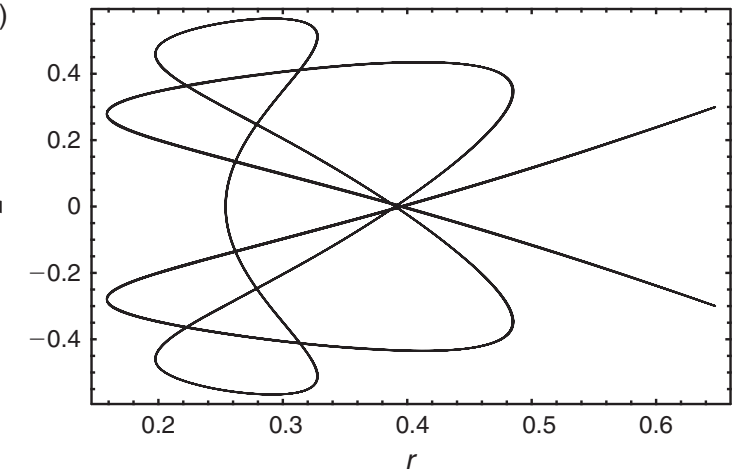

(g)

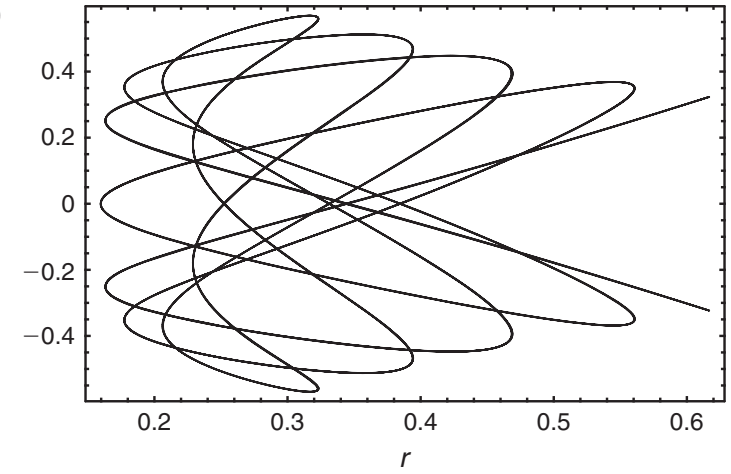

(b)

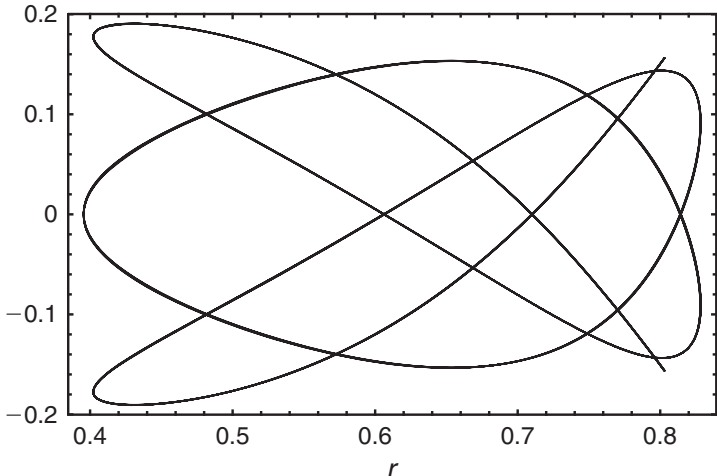

(d)

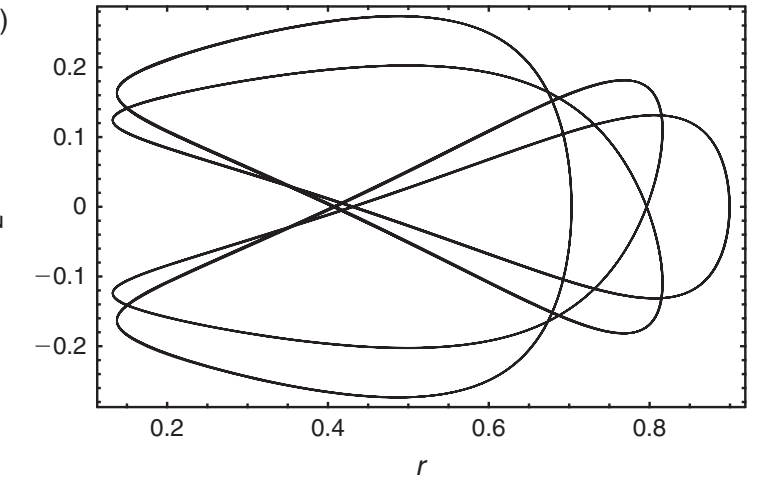

(f)

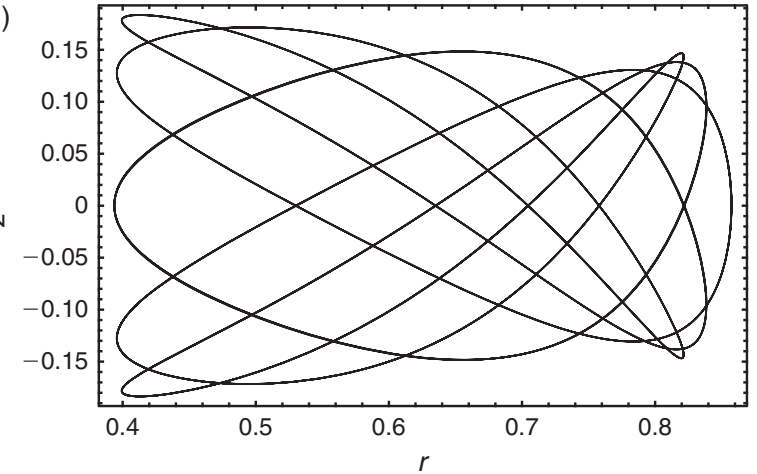

(h)

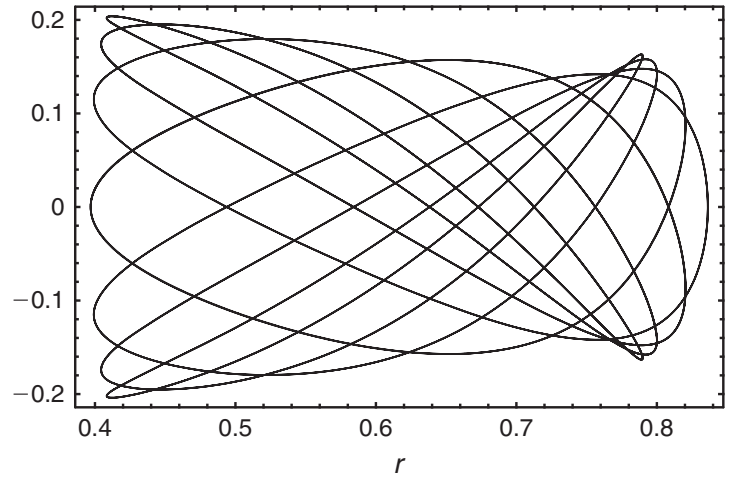

Figure 7 Secondary resonant periodic orbits of the dynamical system. The initial conditions of the orbits are given in the text.

Our numerical calculations suggest that all regular orbits cannot escape from the dynamical system and therefore are trapped regular orbits. We have tested more than 1000 regular orbits with different and random initial conditions $\left(r_{0}, z_{0}=0, p_{r 0}\right)$ in each phase plane (for different values of energy $h$ and angular momentum $L_{z}$ ). In all cases, the regular orbits (basic orbits and also orbits corresponding to secondary resonances) are trapped, regardless of the total integration time interval, and they remain inside the open $\mathrm{ZVC}$.

What is much more interesting is the behavior of the chaotic orbits of the dynamical system. The unified chaotic sea shown in the $\left(r, p_{r}\right)$ phase plane of Figure 4(a) is produced by a single chaotic orbit. This orbit has 

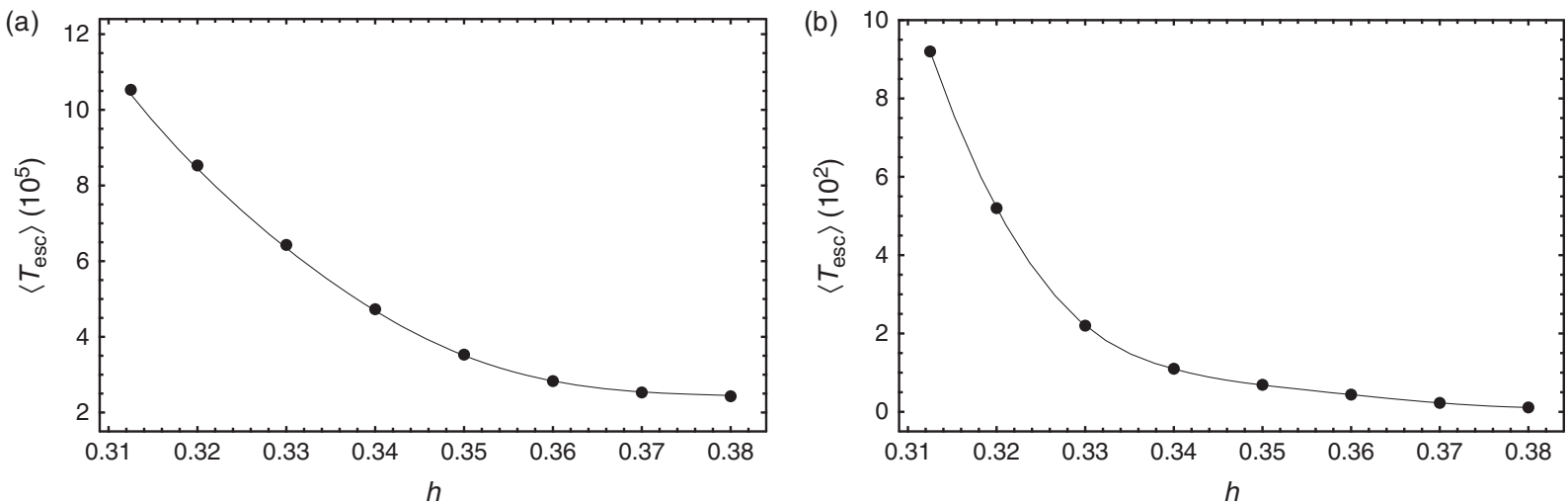

Figure 8 The evolution of the mean value of the escape period $\left\langle T_{\text {esc }}\right\rangle$ for each value of energy $h$ for (a) trapped chaotic orbits and (b) fast-escaping chaotic orbits.

initial conditions $r_{0}=0.48, z_{0}=0, p_{r 0}=0$, while the value of $p_{z 0}$ is found from the energy integral (4). This orbit escapes, after spending a time interval of more than $9 \times 10^{5}$ time units $\left(9 \times 10^{12} \mathrm{yr}\right)$ until it finds the escape channel to the open ZVC. On the other hand, recent evidence from the Hubble key project and measurements of fundamental cosmological parameters from the Wilkinson Microwave Anisotropy Probe (WMAP: Komatsu et al. 2011) give an estimation for the age of the Universe of $(1.375 \pm 0.13) \times 10^{10} \mathrm{yr}$. As the time needed for the chaotic orbit to escape $T_{\text {esc }}$ is much larger than the age of the Universe, we can consider it as a 'nonescaping orbit'. Going one step further, we can say that this orbit is an example of a non-escaping orbit, more specifically a trapped chaotic orbit, that is responsible for the phenomenon of 'trapped chaos' in galactic systems with energies larger than the energy of escape. The unified chaotic region shown in the phase plane of Figure 4(b) is also produced by another trapped chaotic orbit. The initial conditions of this orbit are $r_{0}=0.57$, $z_{0}=0, p_{r 0}=0$. This orbit can also be considered as an 'non-escaping' orbit, as it escapes to infinity after spending a total time interval of more than $7 \times 10^{5}$ time units. Moreover, the chaotic sea shown in the phase plane of Figure 4(c) is also a product of a trapped chaotic orbit, with initial conditions $r_{0}=0.625, z_{0}=0, p_{r 0}=0$. In this case, the escape period of this orbit is about $4 \times 10^{5}$ time units. Similarly, the confined chaotic layer shown in the phase plane of Figure 4(d) is also produced by a trapped chaotic orbit. This orbit has initial conditions $r_{0}=0.512$, $z_{0}=0, p_{r 0}=0$, while its escape period is about $3 \times 10^{5}$ time units. We observe that as the value of energy $h$ increases the escape period of the trapped orbits decreases, but in all cases the value of $T_{\text {esc }}$ is at least about 100 times larger than the age of the Universe!

Extensive numerical calculations suggest that about $62 \%$ of the total tested chaotic orbits stay inside the ZVC before escaping, for time intervals larger than the age of the Universe. In order to study this behavior in more detail, we have checked the escaping period $T_{\text {esc }}$ for 1000 orbits for seven pairs of values: $h=h_{\mathrm{esc}}, L_{z}=L_{z c}-\Delta L$ with $\Delta L=0.01$, that is 7000 orbits. Therefore, we can say that our numerical experiments indicate that about $62 \%$ of chaotic orbits are trapped chaotic orbits. Figure $8(\mathrm{a})$ shows a plot describing the evolution of the mean value of the escape period $\left\langle T_{\text {esc }}\right\rangle$ for each value of the energy $h$. In this case, the mean values of the escape periods refer to the trapped orbits, which represent $62 \%$ of the total chaotic orbits. One can observe that the mean value of the escape period decreases rapidly as the value of the energy increases. Moreover, for large values of the energy $(h \geq 0.35)$ the slope is small and the curve tends to become horizontal. As we have discussed earlier, one single orbit covers the entire chaotic domain in each panel of Figure 4. However, if we start from a different initial condition $\left(r_{0}, p_{r 0}\right)$ in the chaotic region of each phase plane, we again cover the entire phase plane but there is a difference. All chaotic orbits with different initial conditions each cover the entire phase plane, but not all of them have the same escape period. This is the basic reason why we have estimated the mean value of the escape period in each case. Moreover, our calculations revealed that the remaining $38 \%$ of the total tested chaotic orbits escape from the open ZVC within very short time intervals. These are fast-escaping chaotic orbits. In Figure 8(b) we can see a plot depicting the evolution of the mean value of the escape period $\left\langle T_{\text {esc }}\right\rangle$ for each value of the energy $h$. In this case, the mean values of the escape periods refer to fast-escaping orbits, which represent $38 \%$ of the total chaotic orbits. One can observe that the mean value of the escape period decreases much more rapidly than in Figure 8 (a) as the value of the energy $h$ increases. Furthermore, for values of energy $(h \geq 0.34)$ the slope is very small and the fitting curve tends to become horizontal. The main conclusion from our orbital analysis is that as the initial conditions $\left(r_{0}, z_{0}=0, p_{r 0}\right)$ of an orbit increasingly approach the critical boundary of the Lyapunov orbit, the value of the escape period $T_{\text {esc }}$ decreases dramatically.

Figure 9 shows four escaping chaotic orbits with different escape periods. Figure 9(a) shows a chaotic orbit with initial conditions $r_{0}=0.92, z_{0}=0, p_{r 0}=0$, while the value of $p_{z 0}$ is always found from the energy integral (Equation 4). This orbit was calculated for a time period of 
(a)

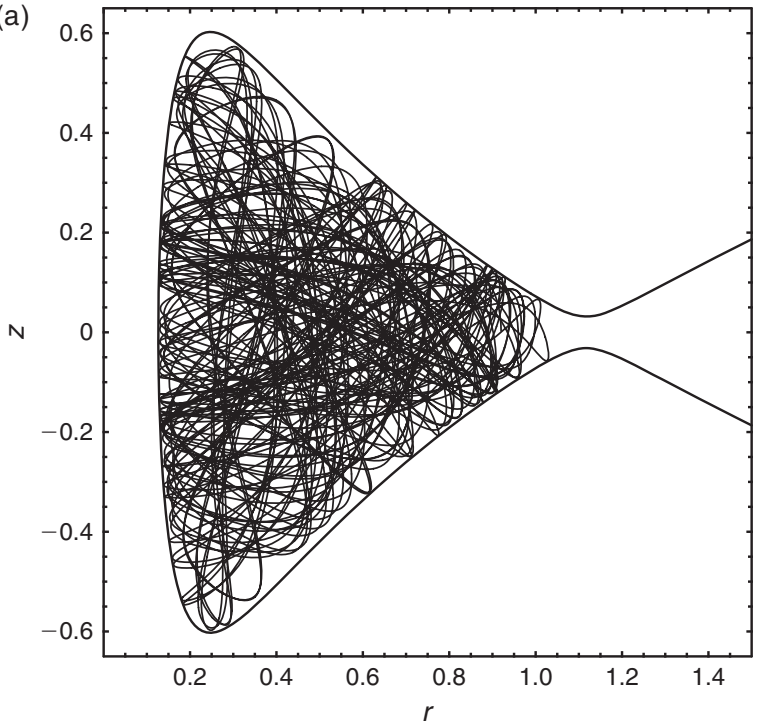

(c)

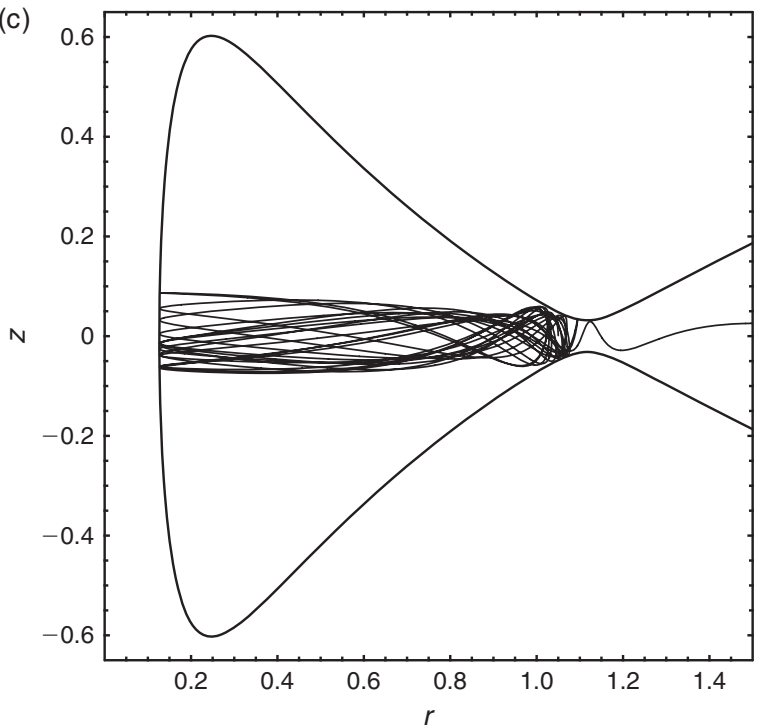

(b)

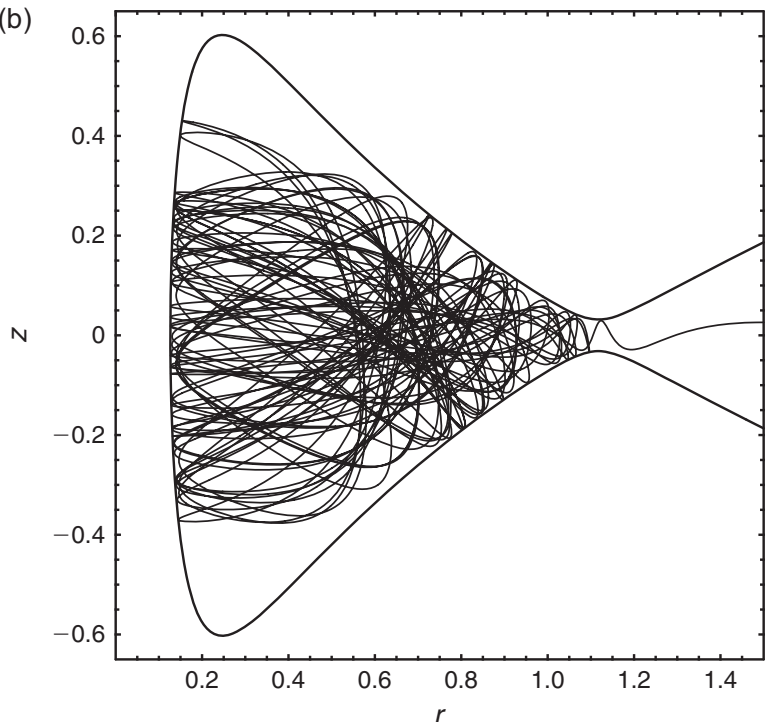

(d)

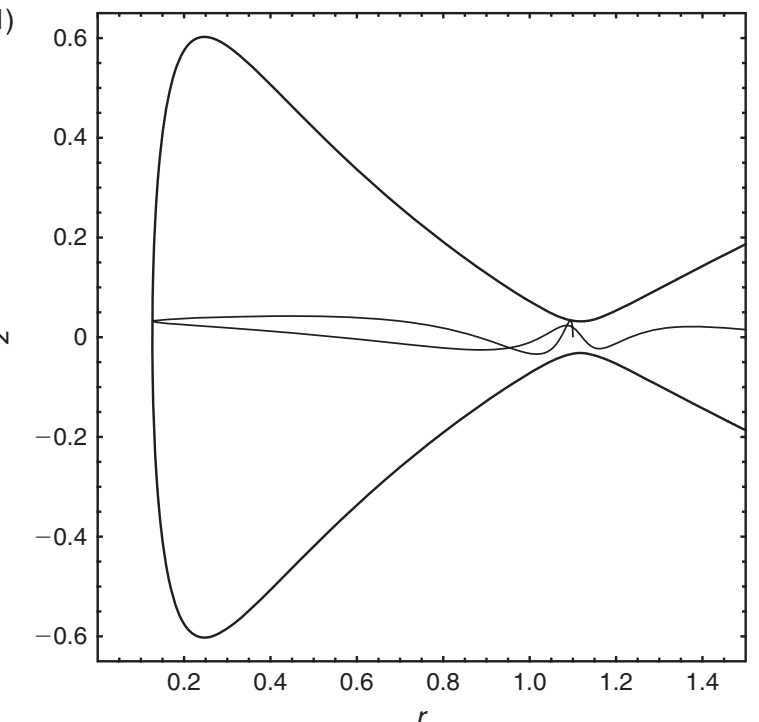

Figure 9 Four escaping chaotic orbits of dynamical system with different periods of escape. (a) A chaotic orbit calculated for 400 time units, (b) the same chaotic orbit as in Figure 9(a), but for the interval between 1450 and 1700 time units, (c) another escaping orbit with escape period of 85 time units and (d) a very fast-escaping orbit with an escape period of 11 time units. The initial conditions of the orbits are given in the text.

400 time units. As we can see, the orbit fills almost all the area inside the ZVC but it does not escape. This orbit escapes after $T_{\mathrm{esc}}=1700$ time units. Figure 9(b) shows the same orbit as in Figure 9(a) but here the orbit was plotted for the the last 250 time units, that is the interval between 1450 and 1700 time units. In this case, the orbit after spending 1700 time units inside the ZVC finally finds the channel and escapes to infinity. In Figure 9(c) we can observe one more escaping orbit with initial conditions $r_{0}=1.05, z_{0}=0, p_{r 0}=0$. This orbit was calculated for only 85 time units. The escape period of this orbit is considerably small. Perhaps we can give an explanation for this behavior by looking at the shape of the orbit given in Figure 9(c). This orbit stays very close to the galactic plane, with small values of $z$ (see Figure 10(b)). Moreover, the orbit is almost parallel to the channel of escape and therefore the test particle can very quickly find its way out from the ZVC. Figure 9(d) shows a very fast-escaping chaotic orbit, with initial conditions $r_{0}=1.1, z_{0}=0$, $p_{r 0}=0$. This orbit escapes from the ZVC after only 11 time units. The values of energy $h$ and angular momentum $L_{z}$ for the orbits shown in Figure 9 are 0.32 and 0.10 respectively.

Figure 10 shows the evolution of the $z$ component of the orbits shown in Figure 9(a) and (c). Looking at Figure 10(a), we observe that the test particle (star) spends long time intervals before it escapes from the $\mathrm{ZVC}$ at high values of $z$. In contrast, things are quite different in Figure 10(b), where we can see that for this orbit the star stays near to the galactic plane with low values of $z$. Therefore, as the star moves almost parallel to the escape channel, it finds the gap in the open ZVC relatively quickly and as a result its escape period is very small. Thus, it seems that there is a relation between the escape period $T_{\text {esc }}$ and the $z$ component of the orbits. In an effort to explore this correlation further, we have calculated the 
(a)

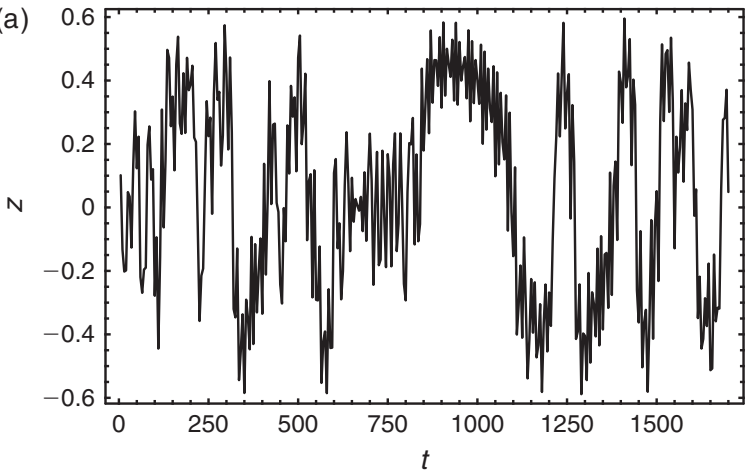

(b)

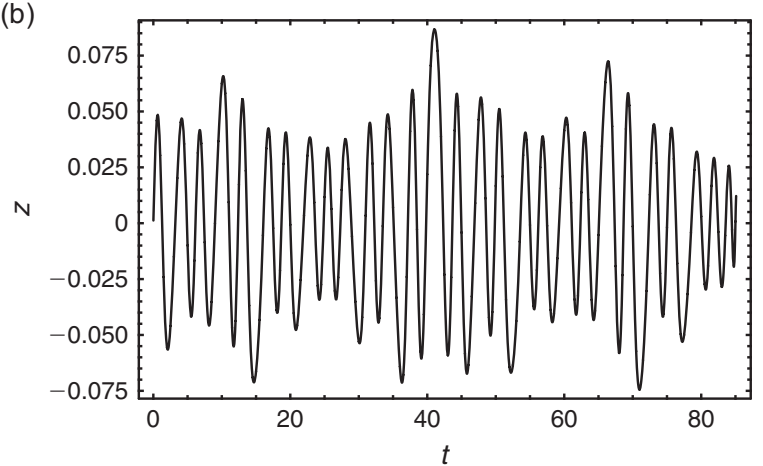

Figure 10 Evolution of $z$ height of a star with time for the orbits shown in (a) Figure 9(a) and (b) Figure 9(c).
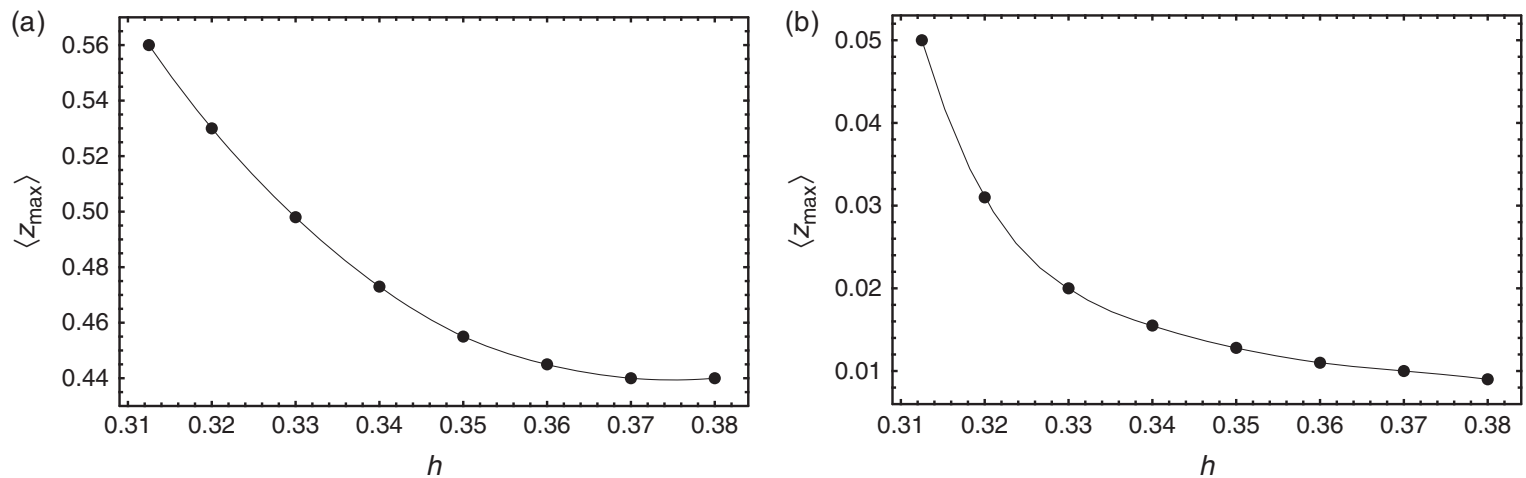

Figure 11 Evolution of the average value of the $z$ component $\left\langle z_{\max }\right\rangle$ for each value of energy $h$ for (a) trapped chaotic orbits and (b) fast-escaping chaotic orbits.

average maximum value of the $z$ component, $\left\langle z_{\max }\right\rangle$, of 100 chaotic orbits (trapped and escaping) for each value of energy $h$. Figure 11(a) depicts a plot describing the evolution of the average value of the maximum $z$ component, $\left\langle z_{\max }\right\rangle$, for each value of energy $h$. In this case, the average value $\left\langle z_{\max }\right\rangle$ corresponds to trapped chaotic orbits. One may observe that the average value $\left\langle z_{\max }\right\rangle$ decreases as the value of energy increases. Similarly, in Figure 11(b) we can see the same plot but in this case corresponding to fast-escaping orbits. Once more, the average value $\left\langle z_{\max }\right\rangle$ decreases as energy $h$ increases. Here, we must point out that in the case of fast-escaping orbits the decrease is much more rapid than in the case of the trapped orbits shown in Figure 11(a). From the plots of Figure 11, we can justify and also explain the behavior of the escape period that was discussed in Figure 8. As the average value $\left\langle z_{\max }\right\rangle$ decreases in both cases (trapped and fast-escaping orbits), this should explain the decrease in the escape period shown in Figure 8. In particular, the patterns in Figure 11 are very similar to those in Figure 8. Moreover, we see that in the case of fast-escaping orbits (Figure 11(b)) $\left\langle z_{\max }\right\rangle$ has much lower values than in the case of trapped chaotic orbits (Figure 11(a)). In Figure 11(b) in particular, the values of $\left\langle z_{\max }\right\rangle$ are 10-40 times smaller than those in Figure 11(a). This means that fastescaping orbits of the dynamical system move very close to the galactic plane, with very low values of the $z$ component, almost parallel to the escape channel of the open ZVC. Consequently, these fast-escaping orbits can find the exit gap easily and much more quickly and therefore this explains their small values of escape period. In contrast, our numerical calculations suggest that the trapped chaotic orbits of the dynamical system have much larger average values of $\left\langle z_{\max }\right\rangle$. Thus, these orbits must spend vast time intervals inside the ZVC (much larger than the age of the Universe) before they eventually find the exit and escape. Therefore, we consider these chaotic orbits as trapped chaotic orbits.

It would be of particular interest to have a better estimation of the degree of chaos for the chaotic orbits in each case. For this purpose, we have computed the Lyapunov characteristic exponent (LCE; see Lichtenberg \& Lieberman 1992 for details). Figure 12(a) shows the LCE for the orbit producing the chaotic sea shown in Figure 4(c). The orbit stays inside the ZVC for about $4.2 \times 10^{12} \mathrm{yr}$ before escaping to infinity. The LCE of this orbit is of order unity. Thus, we are in the case of 'fast chaos' (see Caranicolas 1990 and references therein). Figure 12(b) shows the time evolution of the LCE of the orbit producing the chaotic region shown in Figure 4(d). Here the orbit escapes after a time period of about $3.2 \times 10^{12} \mathrm{yr}$. In this case, we again have fast chaos but the value of the LCE is much larger. Figure 13 shows a plot of the relation between the average value of the LCE and the energy $h$. Here we must point out that, in general, different chaotic orbits have different values of LCE. As we have regular regions in all cases and only one unified chaotic domain in each $\left(r, p_{r}\right)$ phase plane of Figure 4 , we 

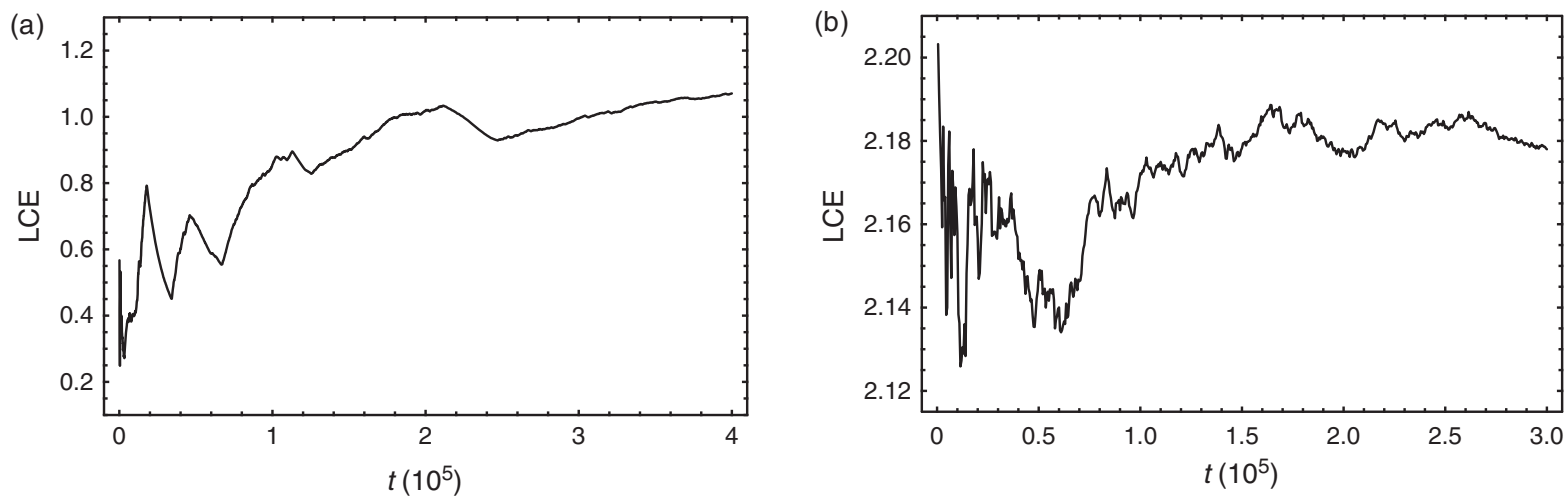

Figure 12 Evolution of the the LCE for the trapped chaotic orbits producing the chaotic regions in (a) Figure 4(c) and (b) Figure 4(d).

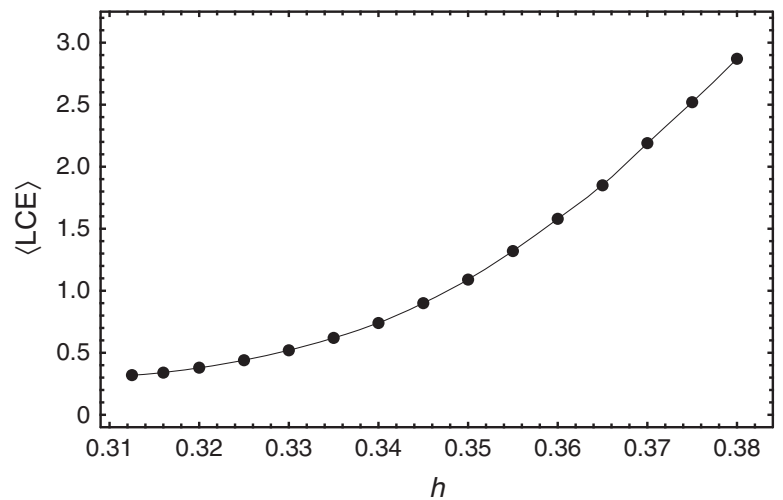

Figure 13 A plot of the relation between the mean value of the LCE and the energy $h$.

calculate the average value of the LCE by taking 1000 orbits with different and random initial conditions $\left(r_{0}, p_{r 0}\right)$ in the chaotic sea (see Caranicolas \& Zotos, 2011a). Note that all calculated LCEs were different at the fifth decimal place in the same chaotic region. Therefore, the mean value of the LCE is a representative value of the degree of chaos for each value of energy $h$. Here we must point out that the obtained average value of the LCE was calculated by choosing both trapped and fast-escaping chaotic orbits. The exact nature of the escape of the chaotic orbit (fast or not) does not add any particular difference to the value of the LCE; therefore we do not need two different diagrams considering trapped and fastescaping chaotic orbits separately, as we used in Figure 8 in order to describe the escape period. In all cases, the values of energy $h$ and angular momentum $L_{z}$ are chosen as described in Section 2. We observe that $\langle\mathrm{LCE}\rangle$ increases drastically as the value of energy increases. In all cases the ZVC is open, except in the case when $h=0.3125$, where the value of $L_{z}=0.01$ was taken. This was done because for this value of the energy, for all values of $L_{z}$, the $\mathrm{ZVC}$ is closed.

If we combine the results from the phase planes shown in Figure 4 together with the plot shown in Figure 13, we can conclude that as the values of energy $h$ and angular momentum $L_{z}$ increase the chaotic region in the phase plane decreases. At the same time, however, as we can see from Figure 13 the degree of chaoticity also increases drastically. Therefore we can say that as the chaotic domain is reduced, this has the result of increasing the degree of chaos. In other words, the more confined a chaotic region is in the phase plane, the stronger the degree of chaos in this region.

\section{Discussion and Conclusions}

In the present article, we have investigated the nature of the orbits of stars in a time-independent galactic-type potential. This potential can be considered to describe the motion in the meridian $(r, z)$ plane near the central parts of an axially symmetric galaxy. Our gravitational model is valid only when $r \leq 1.5$. The quartic terms in Equation 1 become predominant as soon as we go beyond the above limit and therefore the model becomes unrealistic and unphysical. Using the conserved component of the angular momentum $L_{z}$, the three-dimensional motion can be confined and studied in the meridian $(r, z)$ plane, which rotates differentially at an angular velocity given by Equation 5.

We studied the phenomenon in which stars escape from the central parts of a galaxy. This phenomenon has been an active field of research over the last decades. Contopoulos (1990), using a simple model with two degrees of freedom and with energies larger than the escape energy, found that most orbits of the system escape quickly but there are also orbits that escape only after an arbitrarily long time or do not escape at all. Similarly, Contopoulos and Kaufmann (1992) studied the nature of orbits in a Hamiltonian that has four channels of escape. They located the 'basins' of escape towards different directions in the cases of fast or slow escape, for various values of the perturbation parameter. Moreover, the work of Kandrup et al. (1999) summarizes a study of the problem of escapes of energetically unbound orbits in strongly non-integrable Hamiltonian systems of two degrees of freedom as an example of phase-space transport in complex systems. This work has led to two significant conclusions. (i) When evolved into the future, ensembles of orbits of fixed energy often exhibit a rapid approach towards a constant escape probability $P_{0}$, the 
value of which is independent of the details of the ensemble and exhibits interesting scaling behavior. Moreover, the values of the critical exponents appear to be relatively insensitive to the choice of Hamiltonian. (ii) At later times, the escape probability decreases in a fashion which, for at least one model system, is well fitted by a power law. This non-trivial time-dependence is attributed to the fact that the possibility of escape to infinity is controlled by the cantori, which can trap chaotic orbits near regular regions for extremely long times. Fukushige \& Heggie (2000) modeled a cluster as a smooth potential plus the steady tidal field of the Galaxy. In this model there is a minimum energy below which stars cannot escape. Above this energy, however, the time-scale on which a star escapes varies with the orbital parameters of the star (mainly its value of energy). This time-scale was quantified and estimated with both theoretical arguments and computer simulations. Within the limitations of the model, it was shown that the time-scale is long enough to complicate the interpretation of full $N$-body simulations of clusters and that stars above the escape energy may remain bound to the cluster for about a Hubble time. A detailed study regarding the dynamics of the outer parts of barred galaxies beyond corotation was made by Contopoulos \& Patsis (2006). They found that in the outer regions of barred galaxies beyond corotation there are three types of orbits: (1) ordered (periodic or quasiperiodic), (2) chaotic, and (3) escaping. Papadopoulos \& Caranicolas (2007) explored the character of orbits in a 'bare' Seyfert 1 dynamical model, when the external perturbation is strong enough to have open ZVCs. In this model the majority of orbits escape to infinity but there are also orbits that are trapped and do not escape at all. Thus, it is evident that our results coincide with the outcomes of previous related work, pointing out that in a Hamiltonian system with escape channel there are two kinds of orbits: (i) escaping orbits and (ii) trapped orbits.

In the current research, we focused our study on the behavior of orbits when the ZVC of the dynamical system is open and therefore orbits can escape to infinity. This happens when $h>h_{\text {esc }}$, the case in which stars hold values of energy larger than the energy of escape. In this work we studied the possibility of escape in a dynamical system with two degrees of freedom and obtained quite striking results. Our results could be compared with outcomes from other studies about the non-integrability of the $J_{2}$ problem (Irigoyen \& Simó 1993) or the central manifolds and destruction of Kolmogorov-Arnold-Moser (KAM) tori in the planar Hill's problem (Simó \& Stuchi 2000).

Using the fixed values of the parameters given in Section 2, we obtained Equation 7, which connects the angular momentum and the energy of escape. Our numerical calculations show that all tested regular orbits do not escape at all from the system. When we refer to regular orbits, we mean both the basic orbits of the system and orbits corresponding to secondary resonances. Thus, we conclude that these orbits are trapped regular orbits. Moreover, the percentage of regular orbits increases as the values of energy $h$ and angular momentum $L_{z}$ increase. More correctly, the whole area of regular orbits in the $\left(r, p_{r}\right)$ phase planes increases as we proceed to higher values of energy. At the same time, the area in the same phase planes corresponding to chaotic orbits decreases. Note that in all cases studied the ZVC is open and the values of the pairs $\left[h, L_{z}\right]$ were chosen using the algorithm described in Section 2.

Our numerical experiments suggest that, in addition to the regular orbits, for a particular pair of $\left[h, L_{z}\right]$ there are also two kinds of chaotic orbits: chaotic orbits that spend large time intervals inside the ZVC before they escape and chaotic orbits that escape very quickly through the escape channel. It was observed that about $62 \%$ of the total tested chaotic orbits stay inside the ZVC for time intervals that are at least 100 times larger than the age of the Universe. Therefore these orbits can be considered as non-escaping. On this basis, we are experiencing a phenomenon of 'trapped chaos', the situation when we have chaotic orbits that are trapped inside an open ZVC. The remaining 38\% of the tested chaotic orbits correspond to orbits that have small escape periods and therefore can be regarded as fast-escaping orbits. The main characteristic of these orbits is that they have initial conditions very close to the critical boundary of the Lyapunov orbit. This is the boundary that orbits should be able to penetrate in order to escape to infinity. Here, we must point out that the fact that orbits with initial conditions very close to the Lyapunov orbit escape quickly is not a necessary condition for fast escape in general (e.g. in Figure 9(d) we may consider the orbit starting close to the left boundary of the ZVC, far from the Lyapunov orbit, and this orbit escapes quickly). The numerical experiments indicate that the average value of the escape period of chaotic orbits (trapped or fast-escaping) strongly depends on the average value of the maximum $z$ component of the orbits. Our main conclusion is that eventually all chaotic orbits, sooner or later, will escape from the system.

In order to have a better estimation of the degree of chaos for the chaotic orbits in each case, we computed the average value of the LCE. Our results indicate that as the chaotic region is reduced the degree of chaos increases as a result. In other words, the more confined a chaotic region is, the stronger the degree of chaos in this region. Especially in the case of trapped chaotic orbits, we deal with 'trapped fast chaos', as in most cases the value of the computed LCE is much larger than unity. When we state that the LCE has a value much larger than unity, we mean that the LCE is larger than $10^{-7} \mathrm{yr}^{-1}$, which is the inverse value of the time unit.

As the phenomenon of trapped chaotic orbits is of particular interest, we shall stay on the subject with some further comments. In the case of trapped regular orbits, for values of energy larger than the energy of escape one could say that these regular trapped orbits may have an additional third integral of motion, which keeps them inside the open ZVC forever. Of course, this cannot be 
mentioned in the case of trapped chaotic orbits, because chaotic orbits cannot have any further integral of motion. What remains, therefore, is a kind of 'stickiness' (see Karanis \& Caranicolas 2002), with a time for the sticky period larger than the age of the Universe!

We consider the outcomes of the present research to be an initial effort, in order to explore the orbital structure of this interesting dynamical system in extensive detail in a future paper. As the results are positive, further investigation will be initiated in order to explore all the available phase space and also to cover the whole range of values of the main involved parameters, such as the energy $h$ and angular momentum $L_{z}$.

\section{Acknowledgments}

The author expresses his thanks to the anonymous referee for a careful reading of the manuscript and also very useful suggestions and comments, which improved greatly the quality and clarity of the present paper.

\section{References}

Anosova, J. P., 1986, Ap\&SS, 124, 217

Benet, L., Trautmann, D. \& Seligman, T. H., 1996, CeMDA, 66, 201

Benet, L., Seligman, T. H. \& Trautmann, D., 1998, CeMDA, 71, 167

Bleher, S., Grebogi, C., Ott, E. \& Brown, R., 1988, PhRvA, 38, 930

Caranicolas, N. D., 1990, A\&A, 227, 54

Caranicolas, N. D., 2001, JA\&A, 22, 309

Caranicolas, N. D. \& Innanen, K. A., 1991, AJ, 102, 1343

Caranicolas, N. D. \& Papadopoulos, N. J., 2003a, A\&A, 399, 957

Caranicolas, N. D. \& Papadopoulos, N. J., 2003b, JA\&A, 24, 85

Caranicolas, N. D. \& Vozikis, Ch. L., 1999, A\&A, 349, 70

Caranicolas, N. D. \& Vozikis, Ch. L., 2002, MeReC, 29, 91

Caranicolas, N. D. \& Zotos, E. E., 2010, AN, 331, 330

Caranicolas, N. D. \& Zotos, E. E., 2011a, RAA, 11, 811

Caranicolas, N. D. \& Zotos, E. E., 2011b, RAA, 11, 1449
Churchill, R. C., et al., 1979, in Como Conference Proceedings on Stochastic Behavior in Classical and Quantum Hamiltonian Systems, Volume 93, Lecture Notes in Physics, ed. G. Casati \& J. Fords (Berlin: Springer), 76

Contopoulos, G., 1990, A\&A, 231, 41

Contopoulos, G., 2002, Order and Chaos in Dynamical Astronomy (Berlin: Springer)

Contopoulos, G. \& Efstathiou, K., 2004, CeMDA, 88, 163

Contopoulos, G. \& Harsoula, M., 2005, NYASA, 1045, 139

Contopoulos, G. \& Kaufmann, D., 1992, A\&A, 253, 379

Contopoulos, G. \& Patsis, P. A., 2006, MNRAS, 369, 1039

Contopoulos, G., Hénon, M. \& Lynden-Bell, D., 1973, in Dynamical Structure and Evolution of Stellar Systems, Lectures of the 3rd Advanced Course of the Swiss Society of Astronomy and Astrophysics, ed. G. Contopoulos, M. Hénon \& D. Lynden-Bell (Sauverny: Geneva Observatory), 91

Deprit, A. \& Elipe, A., 1991, CeMDA, 51, 227

Elipe, A., 2001, Math. Comp. Sim., 57, 217

Elipe, A. \& Deprit, A., 1999, MeReC, 26, 635

Fukushige, T. \& Heggie, D. C., 2000, MNRAS, 318, 753

Irigoyen, M. \& Simó, C., 1993, CeMDA, 55, 281

Kalnajs, A. J., 1973, PASA, 2, 174

Kandrup, H., Siopis, Ch., Contopoulos, G. \& Dvorak, R., 1999, Chaos, 9, 381

Karanis, G. I. \& Caranicolas, N. D., 2002, AN, 323, 3

Komatsu, E., et al., 2011, ApJS, 192, 18

Lichtenberg, A. J. \& Lieberman, M. A., 1992, Regular and Chaotic Dynamics (2nd ed.; Berlin: Springer)

Maciejewski, W. \& Athanassoula, E., 2007, MNRAS, 380, 999

Papadopoulos, N. J. \& Caranicolas, N. D., 2007, A\&AT, 26, 301

Romero-Gómez, M., Athanassoula, E., Antoja, T. \& Figueras, F., 2011, MNRAS, 418, 1176

Simó, C. \& Stuchi, T. J., 2000, PhyD, 140, 1

Siopis, Ch., Kandrup, H., Contopoulos, G. \& Dvorak, R., 1995a, NYASA, 773, 221

Siopis, Ch., Contopoulos, G. \& Kandrup, H., 1995b, NYASA, 751,205

Siopis, Ch., et al., 1996, CeMDA, 65, 57

Tremaine, S. D. \& Weinberg, M. D., 1984, MNRAS, 209, 729

Zotos, E. E., 2011a, NewA, 16, 391

Zotos, E. E., 2011b, CSF, 44, 501 